\title{
Niveles de Progresión de Gestión de la Convivencia Escolar a Nivel Intermedio en Chile
}

\section{Management Levels of School Coexistence at a Middle Level in Chile}

\author{
Paula Ascorra ${ }^{1,2, \star}$, Karen Cárdenas ${ }^{1,2}$ y Javier Torres-Vallejos ${ }^{1,3}$ \\ ${ }^{1}$ Centro de Investigación para la Educación Inclusiva, Chile \\ ${ }^{2}$ Pontificia Universidad Católica de Valparaíso, Chile \\ ${ }^{3}$ Universidad Andrés Bello, Chile
}

\section{DESCRIPTORES:}

Administración escolar

Nivel intermedio

Nivel distrital

Convivencia escolar

Conglomerados

\begin{abstract}
RESUMEN:
La crisis mundial generada por el COVID-19 ha tenido diversas repercusiones a nivel global. Esta situación nos invita a reflexionar sobre nuestra institucionalidad y la importancia de la escuela dentro de los procesos de inestabilidad. En este aspecto, la gestión escolar a nivel intermedio o distrital (sostenedores en Chile) se realza como un agente clave en la articulación territorial y atención a necesidades de cuidado con sus comunidades escolares. En este artículo se propuso conocer las modalidades de gestión en convivencia escolar existentes en sostenedores chilenos. Mediante un análisis de conglomerados bietápico y un árbol de decisiones, se identificaron grupos de sostenedores de acuerdo con su gestión de la convivencia escolar. Los resultados muestran la presencia de tres niveles de gestión, cuyos abordajes varían de menor a mayor complejidad. Estos grupos se diferencian según características como la dependencia (pública o subvencionada), cantidad de escuelas a cargo, conocimiento del territorio y reconocimiento del logro en convivencia. Asimismo, se identifica como central la socialización de roles y funciones de estos encargados. Se discute la necesidad de evaluar las condiciones político-institucionales que afectan el abordaje y gestión en convivencia escolar a nivel intermedio, lo que resulta crucial en situaciones de crisis.
\end{abstract}

\section{KEYWORDS:}

School management

Middle level

District level

School coexistence

Cluster

\section{ABSTRACT:}

The world crisis generated by COVID-19 has had various repercussions at the global level. This situation invites us to reflect on our institutionality and the importance of the school within the processes of instability. In this aspect, school management at the middle or district level (sostenedores/supporters in Chile) stands out as a key agent in territorial articulation and attention to care needs with their school communities. This article intends to know the management modalities in school coexistence existing in Chilean supporters. Through a two-stage cluster analysis and a decision tree, groups of supporters were identified according to their management of school coexistence. The results show the presence of three levels of management, whose approaches vary from less to more complexity. These groups are differentiated according to characteristics such as dependency (public or subsidized), number of schools in charge, knowledge of the territory, and recognition of achievement in coexistence. Likewise, the socialization of roles and functions of these managers is identified as central. The need to evaluate the political-institutional conditions that affect the approach and management of school coexistence at an intermediate level, which is crucial in crisis situations, is discussed.

CÓMO CITAR:

Ascorra, P., Cárdenas, K. y Torres-Vallejos, J. (2021). Niveles de progresión de gestión de la convivencia escolar a nivel intermedio en Chile. Revista Internacional de Educación para la Justicia Social, 10(1),227-243.

https://doi.org/10.15366/riejs2021.10.1.014

*Contacto: paula.ascorra@pucv.c

ISSN: 2254-3139

revistas.uam.es/riejs
Recibido:

15 de octubre 2020

$1^{\text {a }}$ Evaluación: 13 de enero 2021

2a Evaluación: 19 de marzo 2021

Aceptado: 10 de abril 2021 


\section{Introducción}

En abril de 2020, la Organización Mundial de la Salud (OMS) decretó pandemia debido al contagio masivo producido por el COVID-19. En ausencia de vacunas que frenen la propagación del virus, la OMS instruyó la medida de distanciamiento físico, obligando el cierre de muchas instituciones, entre ellas, la escuela, y recomendando la implementación de procesos de enseñanza y aprendizaje virtual que hagan énfasis en los componentes de contención socioemocional (Reimers y Sychleicher, 2020; UNESCO, 2020).

En el caso de Chile, la situación de crisis sanitaria se ha complejizado aún más, debido a que el país venía enfrentando una crisis social de gran envergadura. El 18 de octubre de 2019 estalla en nuestro país una demanda transversal a distintas clases sociales, partidos políticos, etnias, géneros y edades, que clama por una revisión de la racionalidad neoliberal implementada en dictadura y profundizada en democracia. De acuerdo con Ramírez y otros (2020) esta racionalidad antepone los intereses económicos por sobre las problemáticas sociales; transforma al ciudadano en un cliente, restringiendo sus derechos y erosionando fuertemente la acción colectiva y cohesión social. Esta crisis económica-política-social, se conecta con una serie de movimientos sociales previos, muchos de ellos gestados desde el mundo de la educación.

En este escenario de crisis permanente, la escuela ha tomado un rol central en el cuidado y bienestar de sus comunidades educativas. Distintas organizaciones e investigadores han concordado que, en contextos de crisis, el cuidado de la salud mental y el bienestar socioemocional son aspectos centrales (Kagawa, 2005; Machel, 2001; Tarabini, 2020). La construcción de una comunidad educativa activa, que conoce las necesidades de su territorio, y que favorece el diálogo y la reflexión, instala un sentimiento de pertenencia, que permite recuperar la percepción de apoyo, permitiendo un mejor afrontamiento a las crisis (Universidad de Chile, 2020).

No obstante, ¿es la escuela la única responsable de atender a las necesidades socioemocionales que permiten enfrentar situaciones de crisis? Anderson y otros (2010) han señalado que, en contextos de crisis y cambio, la gestión realizada a nivel intermedio (middle level, district level) es fundamental. Entendemos el nivel intermedio como aquel órgano institucional que se ubica por debajo del Ministerio de Educación de cualquier país y sobre la escuela. En términos concreto corresponde a órganos que por lo general se organizan territorialmente y velan por la educación de un territorio, de un distrito, de una comuna, de un ayuntamiento, de un municipio o de un borought. Los directores de educación distrital o comunal (sostenedores en Chile) son quienes cuentan con los recursos y la institucionalidad para atender a las necesidades de comunidades escolares que adscriben a un mismo territorio.

Particularmente en Chile existe una amplia diversidad de sostenedores o directores de educación. La reforma educacional del ' 81 inauguró la entrada masiva de privados a la educación, quienes podían recibir subvención del Estado vía vouchers y podían lucrar (Assaél et al., 2011). Esto posibilitó la instalación de diferentes modalidades de administración, sujetas a sus fuentes de financiamiento (nulo/ parcial/total), pero también, generó una amplia diversidad en la gestión ante las escasas políticas que delimitaran y/o regularan las funciones del sostenedor (Raczynski et al., 2019).

La evidencia producida en Chile ha demostrado que la gestión de la convivencia escolar ocupa el último lugar de prioridades en los municipios, y que existe una estructura jerárquica verticalista que baja directamente desde el Ministerio de Educación (MINEDUC) a los establecimientos, sin considerar la participación de sostenedores, ni la interacción de éstos con sus comunidades escolares. Así, el rol de los sostenedores, quienes pueden ser los propios alcaldes, Corporaciones, Directores de Educación (DEM o DAEM), o privados, se focaliza más en el cumplimiento de acciones administrativas, que en la mejora de los procesos técnico-pedagógicos (González et al., 2015; Raczynski, 2012; Raczynski, y Salinas, 2006; Román, y Carrasco, 2007).

Atendiendo a estos antecedentes, en que este artículo se propone identificar y caracterizar las modalidades de gestión en convivencia escolar de sostenedores chilenos. Lo anterior resulta crucial, pues existen escasos estudios en materia de convivencia escolar que permitan abordar la amplia diversidad de sostenedores existentes en Chile (Ascorra et al., 2020a; Ascorra et al., 2020b; Gallardo, 2009a; 2009b; Sánchez et al., 2018). Además, es el nivel intermedio quien permitiría propiciar estrategias de 
afrontamiento a las necesidades de las escuelas y sus territorios de manera rápida, oportuna y directa, lo cual se hace aún más relevante en situaciones de crisis (Anderson et al., 2010; Kagawa, 2005).

\section{Gestión del nivel intermedio}

Los sistemas escolares se pueden articular en dos o tres niveles. Un sistema se articula en dos niveles cuando las demandas emanadas desde el Estado -representadas por el Ministerio de educación u otros organismos- recaen directamente sobre las escuelas. Por lo general, esta arquitectura educacional sigue un modelo top down (Hargreaves, 2010), es decir, un primer nivel de políticas públicas que dicta normativas y estándares; $y$, un segundo nivel de la escuela, quien debe cumplirlos. Este modelo es verticalista y deja fuera al sostenedor, actor clave en los procesos de reforma y mejora de la calidad educativa (Campbell y Fullan, 2006).

La investigación internacional sugiere que los procesos de mejora educativa demandan una arquitectura educacional organizada en tres niveles. Esto es, la participación de fuerzas que vienen desde el nivel central, nivel local y de la escuela. Estos modelos articulan sus fuerzas en un doble flujo de información. De manera continua utilizan flujos de información top down y botton up. La información top down permite bajar la política pública y los acuerdos emanados a nivel central hacia el nivel local. La información botton up permite al nivel local de un distrito y/o sus escuelas levantar necesidades y desarrollar estrategias asociadas a su contexto (Campbell y Fullan, 2006; Hargreaves, 2010; Kowalski y Limber, 2007). Raczynski (2012) sostiene que el nivel intermedio funciona como una bisagra entre la base del sistema y el nivel nacional, ayudando a equilibrar las fuerzas que ejercen desde arriba (curriculum, estándares y otros) con las demandas que surgen desde abajo (docencia, desarrollo profesional, competencias y liderazgo directivo, situaciones de crisis, eventos de emergencia como incendios, terremotos, aluviones, entre otros).

La incorporación del sostenedor en la gestión educativa se ha postulado como fundamental para países que poseen sistemas educacionales descentralizados, como el caso de Chile (Delannoy y Guzmán, 2009). Lo anterior debido a que el sostenedor puede rescatar las demandas locales y hacerlas dialogar con la política pública. Concretamente, una buena gestión del sostenedor permitiría: a) aumentar la pertinencia de la política pública; b) potenciar la relación escuela-familia-comunidad; c) movilizar recursos locales para la mejora continua; d) lograr sinergia entre el programa escolar y; e) facilitar el trabajo en red, potenciando el intercambio de experiencias y buenas prácticas entre escuelas (Delannoy y Guzmán, 2009).

En Chile, la implementación de la Ley de Subvención Escolar Preferencial (Ley No. 20.248, 2008), Ley General de Educación (Ley No. 20.370, 2010) y Ley de Sistema Aseguramiento de la Calidad (Ley No. $20.501,2011)$, comienzan a demandar un mayor involucramiento por parte de los sostenedores en los procesos de mejora. De acuerdo con Weinstein y otros (2010), los sostenedores deben velar por el mejoramiento de los resultados de aprendizaje y cumplir sus estándares, aspectos relacionados a un rol técnico-pedagógico de mejora continua (MINEDUC, 2014).

Aun cuando estas políticas proponen nuevas funciones para los sostenedores, no se han desarrollado directrices claras, ni se han instalado capacidades respecto a cómo deben desempeñar su rol (González et al., 2015). Tampoco se ha realizado un adecuado proceso de acompañamiento y/o sensibilización, por lo que no se puede asegurar un cambio en la práctica social con la que ejecutan sus responsabilidades. De acuerdo con Espínola y Silva (2009), la normativa aplicada previamente a los sostenedores excluía funciones técnico-pedagógicas que eran responsabilidad de los Departamentos de Educación Provincial (DEPROV). Debido a ello, por más de 25 años los sostenedores desempeñaron un rol netamente administrativo, funcionado más bien como "cajas pagadoras" u "organismos de empleo" (Raczynski, 2012).

La reforma de 1981 posibilitó la entrada masiva de privados a la educación, quienes podían recibir subvención del Estado vía vouchers y podían lucrar. Esto instaló una amplia heterogeneidad de sostenedores, dificultando -hasta el día de hoy- su caracterización. Es así, como actualmente en Chile podemos distinguir al menos cinco tipos de sostenedores según su dependencia administrativa (Ascorra et al., 2020b): 
- Municipales (SM). De financiamiento público. Representan al 9,3\% de los sostenedores, son responsables del $42 \%$ de los establecimientos y concentran el 34,1\% de la matrícula escolar. Estos establecimientos congregan principalmente a estudiantes de estrato socioeconómico bajo (González, 2017) y tienen una matrícula promedio de 300 estudiantes $(D S=185,5)$ por establecimiento (MINEDUC, 2018).

- Particulares subvencionados (SPS). De financiamiento mixto (público-privado), y que a contar del año 2015 (Ley No. 20.845, 2015) se comienzan a organizar a través de corporaciones y fundaciones sin fines de lucro. Representan al 77,7\% de los sostenedores, son responsables del $48 \%$ de los establecimientos y tienen el 53,9\% de la matrícula. Estos sostenedores concentran en su matrícula a estudiantes de estratos medios (González, 2017) y tienen un promedio de 315 estudiantes $(D S=397,5)$ por establecimiento.

- Particulares (SP). De financiamiento privado. Representan al 12\% del país, son responsables del $9 \%$ de los establecimientos, con un $9,2 \%$ de la matricula. Su matrícula está compuesta mayoritariamente por estudiantes de estrato socioeconómico alto (González, 2017) y poseen en promedio $497(D S=474,8)$ estudiantes por establecimiento.

- Administración delegada (AD). Entidades privadas que administran establecimientos técnico-profesional de financiamiento público. Representan al 1\% de los sostenedores, son responsables del 0,6\% de los establecimientos y concentran un 1,2\% de la matrícula. Estos sostenedores tienen un promedio de 683 estudiantes ( $\mathrm{DS}=213,2)$ por establecimiento.

- Servicios Locales de Educación (SLEP). Nueva institucionalidad instaurada a contar del año 2017 que concentra a establecimientos de distintas comunas. Actualmente representan a un $0,2 \%$ de los establecimientos y tienen un promedio de 258 estudiantes $(D S=129,0)$.

Además de estas características, podemos encontrar otras vinculadas a sus condiciones de gestión. Estas características se presentan principalmente en SM y SPS -quienes poseen mayor cantidad de establecimientos y matricula-. En el caso de SM, encontramos aquellos con una alta o muy alta cantidad de escuelas, baja y/o alta matricula, y con escuelas rurales y/o urbanas. Un estudio realizado por Raczynski (2012), develó que aquellas municipalidades más grandes congregan más recursos, más estudiantes y poseen departamentos de educación más complejos y eficientes. Mientras que, aquellas municipalidades más pequeñas poseen menos recursos y se encuentran menos profesionalizados. Además, Raczynski (2012) identificó que existían municipios con proyectos ambiciosos, que movilizaban a su personal, mientras que otros repetían de manera rutinaria sus prácticas burocráticas-administrativas, sin pretensiones de mejora. En el caso de SPS, podemos encontrar aquellos que poseen a su cargo un solo establecimiento y funcionan como pequeñas empresas familiares, donde el sostenedor es director y dueño del establecimiento, y su equipo son familiares o amigos (Carrasco et al., 2014). Pero también, podemos encontrar aquellos que funcionan en red o que atienden a un grupo de escuelas, en su mayoría de órdenes religiosas.

Donoso-Díaz y otros (2015) refieren que uno de los principales problemas de la heterogeneidad de sostenedores en Chile es lo que se conoce como "desbalance del sistema". El desbalance se produce cuando la normativa es desigual y permite acciones diferenciadas, produciendo condiciones desfavorables para la gestión en algunos sostenedores. Ejemplo de ello son las relaciones contractuales diferenciadas por dependencia. En este aspecto, un estudio realizado por López y otros (2020) constató que los establecimientos municipales tendrían una mayor concentración de profesionales con baja experiencia y mayor cantidad de horas de contratación, mientras que en establecimientos privados y subvencionados existiría una mayor cantidad de profesionales de gran experiencia con contrataciones de baja cantidad de horas. El tipo de contrato, la cantidad y calidad de los profesionales, es considerado un factor relevante para la mejora en convivencia escolar (Ascorra et al., 2020a; Cohen et al., 2009; NSBA, 2009; Raczynski, 2012). Para el caso de Chile esto se hace más relevante, pues son los sostenedores quienes se encargan de gestionar el recurso humano, quienes a su vez tienen escasa responsabilidad en los procesos de mejora continua. 


\section{Gestión de la convivencia escolar}

Entendemos convivencia escolar (CE) como el conjunto de patrones experienciales de la vida en la escuela, que se materializan a través de normas, objetivos, valores, relaciones interpersonales, procesos de enseñanza-aprendizaje y estructura organizacional (Cohen et al., 2009, p. 182). La "Política Nacional de Convivencia Escolar 2015/2018” define convivencia escolar como

un fenómeno social cotidiano, dinámico y complejo, que se expresa y construye en y desde la interacción que se vive entre distintos actores de la comunidad educativa, que comparten un espacio social que va creando y recreando la cultura escolar propia de ese establecimiento. (MINEDUC, 2015, p. 25)

Específicamente, la gestión de la convivencia escolar a nivel intermedio es comprendida como aquellas acciones y apoyos que los sostenedores brindan a sus escuelas para mejorar la calidad y vida dentro las comunidades escolares (Ascorra et al., 2019a). Esta gestión comprende acciones como el diseño, planificación, implementación, evaluación y seguimiento de medidas de promoción, prevención e intervención en convivencia escolar.

La investigación internacional ha identificado distintos modelos de gestión de la CE a nivel intermedio. Cohen y otros (2009) han propuesto una matriz compuesta por cuatro dimensiones de gestión: a) Organización de políticas en clima escolar (liderazgo del nivel intermedio, inclusión de programas focalizados); b) Evaluaciones e instrumentos en clima escolar (instrumentos validados, contextualizados a las necesidades territoriales y con disponibilidad para su uso); c) Definiciones de clima escolar (precisión de la definición y traducción de las medidas) y, d) Asistencia Técnica (contratación de personal, disponibilidad de recursos, intervenciones basadas en la evidencia).

La National School Board Assossiation (NSBA, 2009), concluyó que las funciones claves para la gestión del nivel intermedio se relacionan con: a) Liderazgo compartido, mejora continua y toma de decisiones compartida; b) Capacidad para crear y mantener iniciativas y c) Apoyo para el personal y las escuelas a través de datos e información contextualizada. Asimismo, la NSBA junto con el Center for Public Education (2017) propusieron un modelo de gestión de cuatro fases cíclicas: 1) Determinación de la situación inicial de las escuelas (línea base), que corresponde al reconocimiento de lo que está sucediendo en CE dentro sus escuelas, considerando el apoyo a la construcción, búsqueda y socialización de información producida; 2) Alinear los recursos, que considera la identificación de recursos disponibles en el nivel intermedio y en las escuelas, que permitan propiciar el cambio; 3) Programas y prácticas, que considera la identificación y evaluación de programas de CE vigentes en las escuelas, estableciendo propuestas de mejora; y 4) Monitorear el progreso, que considera la evaluación de logros y obstáculos para el cambio educativo, además de identificar factores y estrategias para su sostenibilidad.

En Chile, Ascorra y otros (2020b) diseñaron y validaron un instrumento de gestión de la convivencia escolar a nivel intermedio compuesto por cuatro dimensiones:

- Planificación estratégica. Considera aquellas acciones que buscan construir un discurso y sentido de la CE a nivel local, que se traduzca en una estrategia coherente entre el nivel intermedio y las escuelas a cargo. Se valora la existencia de una articulación horizontal (las prácticas y planes de mejora de las escuelas son coherentes entre sí) y una articulación vertical (la misión, visión y planes de las escuelas están alineados con los del sostenedor).

- Planificación basada en la evidencia. Considera todas aquellas acciones que involucran la utilización de evidencia empírica sobre la CE producida por las escuelas y el sostenedor. La toma de decisiones se debe realizar a partir de la producción de evidencia empírica para producir transformaciones, así como también debe considerar la socialización y transparencia en la entrega de esta información.

- Recursos financieros. Considera la disponibilidad de recursos económicos en el nivel intermedio para CE. La gestión de la convivencia escolar debe contar con un presupuesto asignado tanto para el sostenedor como para las escuelas y la posibilidad de redistribuirlo de forma autónoma según las necesidades locales. 
- Recursos humanos. Considera las condiciones y características de los profesionales que gestionan la $\mathrm{CE}$ en las escuelas. La literatura indica que la formación, experiencia, tipo de contratación y la cantidad de personal que trabaja en CE impacta en los resultados de mejora. Si bien cada sostenedor tiene sus propias tradiciones y políticas, todos deben avanzar hacia estrategias de formación para su personal (Anderson et al., 2010; NSBA, 2017).

Tras una revisión sistemática realizada por las autoras de este proyecto, en Chile sólo se encontraron tres investigaciones que abordaran directamente la relación entre la gestión de la CE y el nivel intermedio desde las percepciones de sostenedores (Ascorra et al., 2020a; Gallardo, 2009a, 2009b; Sánchez et al., 2018). El resto de las investigaciones sólo lo hace desde su vinculación teórica. El estudio realizado por Gallardo (2009a; 2009b) en cinco municipios de la región metropolitana, mostró una alta congruencia con lo reportado a nivel internacional (Anderson, 2013; Cohen et al., 2009). Dentro de sus principales hallazgos destacan la importancia de la coordinación entre los encargados de los municipios y de las escuelas; la importancia de la formación de los equipos, y los recursos profesionales que dispone el municipio en materia de CE. Los elementos que limitarían las acciones del sostenedor serían la orientación rígida para satisfacer sistemas de accountability, la competencia y la falta de coordinación entre escuelas (Gallardo, 2009a).

Un segundo estudio realizado por Sánchez y otros (2018) en seis municipios mostró resultados similares. Este estudio evidenció que los sostenedores no poseen recursos destinados para CE, tienen escasa formalización de sus equipos, y que estos se encuentran escasamente capacitados. Tampoco se observó un proyecto institucional que apoyara su gestión o una adecuada articulación en red entre establecimientos. A nivel general, se concluyó que la gestión de la CE se encuentra en una fase de instalación, con escasos procesos de gestión, y que estos se encuentran focalizados a la atención de casos críticos y/o el cumplimiento de la normativa vigente. Asimismo, se observó una baja influencia del sostenedor sobre los equipos de convivencia de las escuelas.

Finalmente, la investigación desarrollada por Ascorra y otros (2020) en 110 sostenedores de distintas dependencias del país, identificó algunos factores incidentes en la gestión de la CE. Específicamente este estudio se focalizó en las dimensiones de conocimiento de las escuelas y su territorio, reconocimiento del logro y gestión de redes. Estas dimensiones mostraron ser incididas por condiciones institucionales como la cantidad de horas de contratación, el tamaño de los equipos y la capacitación en convivencia escolar.

En conclusión, estos estudios permiten constatar que es necesario que directores de educación o sostenedores cuenten con modelos de gestión en convivencia escolar que integren factores como: la existencia de un equipo profesional especializado, la implementación de políticas, un sistema de evaluación y seguimiento, y planes atingentes. Asimismo, es necesario que estos modelos sean conocidos y comprendidos por los diferentes miembros de la comunidad escolar. No basta con la creación de acciones a nivel intermedio, estas deben permear a las escuelas y formar parte de sus acciones cotidianas. Estos factores, pueden afectar de manera sustancial el tipo de gestión de la convivencia que se desarrolle, y por consecuencia, pueden tener implicancias directas en la calidad y vida escolar. De esta manera, es necesario que los equipos a nivel intermedio tengan conocimiento de las acciones desempeñadas, de cara a tener un conocimiento especializado de las dimensiones a mejorar.

\section{Método}

El estudio fue transeccional de tipo descriptivo-exploratorio (Hernández et al., 2010).

\section{Participantes}

El muestreo fue estratificado proporcional en función de la dependencia administrativa y la cantidad de establecimientos a cargo del sostenedor, respetando el tamaño del estrato en la población (Canales, 2006). Participaron 519 sostenedores de todas las dependencias del país (20,6\% SM, 70,9\% SPS, 6,6\% $\mathrm{SP}, 1,5 \%$ AD y $0,4 \% \mathrm{SLEP})$. La mayoría mujeres $(56,2 \%)$ a cargo de sólo un establecimiento $(59,9 \%)$. 


\section{Instrumentos}

Se utilizó la escala de gestión de la convivencia escolar a nivel intermedio validada por Ascorra y otros (2020b). Este instrumento se compone de 19 ítems evaluados en una escala Likert de 4 puntos (1: Totalmente en desacuerdo - 4: Totalmente de acuerdo), agrupados en cuatro dimensiones de gestión: Planificación estratégica (cinco ítems - v.g. "Los establecimientos participan en el diseño del plan de CE del sostenedor"), Planificación basada en la evidencia (siete ítems - v.g. "Incentivo el uso de datos e informaciones de los establecimientos para la construcción de sus planes de CE (planes anteriores, IDPS, SIMCE)"), Recursos financieros (cuatro ítems - v.g. "Puedo reasignar recursos financieros de ser necesario"), y Recursos humanos (tres ítems - v.g. "Las horas de dedicación de los encargados de CE de los establecimientos son suficientes para atender sus necesidades"). La escala presenta una alta fiabilidad a nivel global $(\alpha=0,878)$.

Como variables de caracterización se utilizó: 1) dependencia administrativa, 2) cantidad de establecimientos a cargo, 3) cantidad de profesionales en CE del sostenedor, 4) formación en CE, y 5) autopercepción del sostenedor en tres ámbitos de gestión de la convivencia escolar. Estas variables desarrolladas por Ascorra y otros (2020a) corresponden a factores identificados por la literatura internacional como relevantes en la gestión, los cuales se detallan a continuación:

- Conocimiento de las escuelas y su territorio: Referido al conocimiento del sostenedor respecto de las escuelas a su cargo y del entorno en el cual están insertas, a través de información producida por sistemas de medición nacional y/o instrumentos elaborados a nivel local;

- Reconocimiento del logro: Referido al reconocimiento sistemático por parte del sostenedor de los logros en CE alcanzados por sus escuelas; $y$,

- Gestión de redes: Referido a la promoción y conformación de redes entre escuelas, y con otras entidades externas, con foco en el apoyo a problemáticas de CE.

Estas dimensiones a su vez se presentan en un orden creciente, correspondientes a cuatro categorías: a) Etapa Intuitiva, referida a una gestión intermedia que recibe y transmite información producida por sistemas de medición nacional (SIMCE, IDPS, categorías de desempeño u otro) sin un carácter diagnóstico y sin procesamiento contextualizado a las necesidades de las escuelas; b) Etapa Incipiente, donde se recibe y analiza esporádicamente información proporcionada por las escuelas e información producida por sistemas nacionales, estableciendo estrategias focalizadas a las escuelas a cargo; c) Etapa Avanzada, donde se produce, analiza y comunica información de las escuelas estableciendo estrategias y focos de intervención de forma no sistemática; d) Etapa Institucionalizada, donde se produce, analiza y comunica información de las escuelas de forma sistemática, identificando focos de intervención y herramientas que promuevan la mejora de forma global.

\section{Procedimiento}

La escala fue aplicada de forma online. Los participantes fueron invitados a través de correo electrónico, considerando el Directorio Nacional de Sostenedores del año 2018 (MINEDUC, 2018). Se realizó seguimiento vía telefónica para asegurar la recepción de la invitación.

La investigación siguió los principios del Comité de Bioética y Bioseguridad de la institución. Para resguardar aspectos éticos, se incluyó un consentimiento informado previo al acceso al instrumento. Asimismo, se incluyeron tres preguntas respecto de: a) la claridad de la información otorgada, b) aprobación del uso de datos; e, c) interés en la participación.

\section{Análisis de datos}

Se realizaron tres tipos de análisis de manera secuencial. En primer lugar, se realizó un análisis descriptivo, que incluyó el cálculo de medias $(M)$, desviaciones estándar $(D S)$ y correlaciones entre las dimensiones del instrumento.

En segundo lugar, se realizó un análisis de conglomerados bietápico en torno las dimensiones de gestión, para identificar posibles grupos naturales de sostenedores. Esta técnica permite analizar simultáneamente datos categóricos y continuos (McIntyre y Pigram, 1992) de manera de caracterizar, no 
solo a las acciones, sino además otras variables que puedan influir en la aplicación de éstas. En un primer momento, los casos fueron agrupados transitoriamente por medio de un árbol de clasificación considerando variables continuas (dimensiones de gestión y número de equipo) y categóricas (dependencia, autopercepción de la gestión, entre otras), y luego, se confirmó su agrupamiento a través de un procedimiento jerárquico, que permite explorar diferentes soluciones y grupos (Rundle-Thiele et al., 2015). Finalmente, una vez identificado los clústeres, se compararon las variables continuas a través de pruebas ANOVA unifactorial y las variables categóricas a través de pruebas de chi-cuadrado, considerando el estadístico exacto de Fisher en el caso de que al menos el $20 \%$ de las frecuencias esperadas fueran menores de 5 (Pardo y Ruiz, 2005).

En tercer lugar, se utilizó la técnica de árbol de decisiones. Esta técnica permite describir cómo una variable dependiente se comporta en relación con otras variables. Primero, se define un grupo de variables predictivas para una variable-criterio (en este caso, pertenencia al clúster), y se genera una red de nodos que muestra cómo la variable dependiente se comporta en relación con las otras variables (Berlanga et al., 2013). Para esto último, se consideraron las acciones de gestión de la convivencia escolar a nivel intermedio (ítems de cada dimensión). Los datos se analizaron utilizando el software estadístico IBM SPSS Statistics 23.0.

\section{Resultados}

El Cuadro 1 presenta los resultados descriptivos de las dimensiones de gestión. Se observa que las medias oscilan entre $M=3,35$ ( $D S=0,59$ ) en Planificación estratégica y $M=2,80$ ( $D S=0,86)$ en Recursos financieros. Todas las correlaciones son positivas y moderadas, aunque las más bajas se observan entre Recursos financieros y las otras dimensiones.

Cuadro 1

Medias y correlaciones dimensiones de gestión de la convivencia escolar a nivel intermedio

\begin{tabular}{lcccccc}
\hline & M & DS & PE & PBE & RF \\
Planificación estratégica (PE) & 3,35 & 0,59 & - & & \\
Planificación basada en la evidencia (PBE) & 3,23 & 0,57 & $0,749^{* * *}$ & - & \\
Recursos financieros (RF) & 2,80 & 0,86 & $0,424^{* * *}$ & $0,409^{* * *}$ & - \\
Recursos humanos (RH) & 3,17 & 0,62 & $0,648^{* * *}$ & $0,750^{* * *}$ & $0,504^{* * *}$ \\
\hline
\end{tabular}

Nota ${ }^{* * *} \mathrm{p}<0,001$

El análisis de clúster bietápico permitió identificar tres grupos de sostenedores, clasificando al 100\% de los participantes. Al comparar los clústeres través de ANOVA, se observa que el clúster 1 presenta mayor frecuencia en las acciones de gestión que el clúster 2, y sucesivamente este con el clúster 3 . Todas las diferencias son estadísticamente significativas (Cuadro 2).

Al comparar la pertenencia de los clústeres y la dependencia, se observa que más del $20 \%$ de las frecuencias esperadas fueron inferiores a 5, por lo que se utiliza la prueba exacta de Fisher. A través de este estadístico, se concluye que existe asociación entre las variables $(\mathrm{p}<0,001)$. El Cuadro 3 muestra que los sostenedores del clúster 1 y 3 son en su mayoría de dependencia subvencionada, mientras que en el clúster 3 la mayoría de los sostenedores corresponden a dependencia municipal $(73,9 \%)$.

En cuanto a la cantidad de establecimientos a su cargo, se observa que los clústeres 1 y 2 presentan una mayor cantidad de sostenedores a cargo de un establecimiento escolar, mientras que en el clúster 3 prima la administración de dos o más (Cuadro 3). 
Cuadro 2

Comparación entre clústeres según variables continuas (ANOVA unifactorial)

\begin{tabular}{|c|c|c|c|c|c|c|c|c|c|}
\hline & \multicolumn{6}{|c|}{ Clúster } & \multirow{3}{*}{$F(2)$} & \multirow{3}{*}{$\mathbf{p}$} & \multirow{3}{*}{$\eta^{2}$} \\
\hline & \multicolumn{2}{|c|}{$\begin{array}{c}1 \\
(n=187)\end{array}$} & \multicolumn{2}{|c|}{$\begin{array}{c}2 \\
(n=198)\end{array}$} & \multicolumn{2}{|c|}{$\begin{array}{c}3 \\
(n=134)\end{array}$} & & & \\
\hline & $\mathbf{M}$ & DS & $\mathbf{M}$ & $D S$ & $\mathbf{M}$ & $D S$ & & & \\
\hline Planificación estratégica & 3,79 & 0,24 & 3,24 & 0,39 & 2,88 & 0,76 & 148,31 & 0,000 & 0,365 \\
\hline $\begin{array}{l}\text { Planificación basada en la } \\
\text { evidencia }\end{array}$ & 3,74 & 0,26 & 3,04 & 0,28 & 2,81 & 0,67 & 231,13 & 0,000 & 0,473 \\
\hline Recursos financieros & 3,34 & 0,69 & 2,79 & 0,70 & 2,06 & 0,73 & 129,17 & 0,000 & 0,334 \\
\hline Recursos humanos & 3,71 & 0,29 & 3,02 & 0,36 & 2,63 & 0,67 & 249,24 & 0,000 & 0,491 \\
\hline N. equipo & 5,53 & 8,79 & 4,19 & 3,84 & 6,84 & 14,57 & 3,24 & 0,040 & 0,012 \\
\hline
\end{tabular}

Nota . . equipo = Número de personas en el equipo de convivencia escolar a nivel intermedio.

La formación en CE no mostró diferencias en función de los clústeres a nivel de charlas, talleres o asignaturas de pregrado. No obstante, si se mostraron diferencias en la formación a través de cursos, asignaturas de postgrado y diplomados, a favor del clúster 1 y 3 .

Respecto a la autopercepción del conocimiento del territorio, gestión de redes y el reconocimiento del logro, se muestra una asociación entre estas dimensiones y los clústeres. En cuanto a la primera dimensión, se observa que en el clúster 1 los sostenedores se visualizan con un conocimiento del territorio de nivel institucionalizado, en el clúster 2 los sostenedores se perciben en un nivel intuitivo e institucionalizado, y en el clúster 3, los sostenedores se perciben principalmente en un nivel intuitivo. Para la gestión de redes, los clústeres 1 y 2 se auto perciben en un nivel incipiente, mientras que en el clúster 3 se perciben en un nivel intuitivo e institucionalizado. Finalmente, en cuanto al reconocimiento del logro, en todos los clústeres se aprecia que una autopercepción mayormente institucionalizada y avanzada (Cuadro 3).

Cuadro 3

Comparación entre clústeres según variables categóricas (prueba de $X^{2}$ )

\section{Clúster}

\begin{tabular}{|c|c|c|c|c|c|c|c|c|}
\hline & 1 & & & & & & & \\
\hline & $\mathbf{n}$ & $\%$ & $\mathbf{n}$ & $\%$ & $\mathbf{n}$ & $\%$ & $X^{2}$ & \\
\hline \multirow{5}{*}{ Dependencia } & SM & 7 & 3,7 & 1 & 0,5 & 99 & 73,9 & - \\
\hline & SPS & 163 & 87,2 & 175 & 88,4 & 30 & 22,4 & \\
\hline & SP & 12 & 6,4 & 21 & 10,6 & 1 & 0,7 & \\
\hline & $\mathrm{AD}$ & 5 & 2,7 & 1 & 0,5 & 2 & 1,5 & \\
\hline & SLEP & 0 & 0,0 & 0 & 0,0 & 2 & 1,5 & \multirow{3}{*}{$209,78^{* * *}$} \\
\hline \multirow{2}{*}{$\begin{array}{l}\text { Cantidad de estableci- } \\
\text { mientos }\end{array}$} & 1 establecimiento & 127 & 67,9 & 172 & 86,9 & 12 & 9,0 & \\
\hline & $\begin{array}{l}2 \text { o más estableci- } \\
\text { mientos }\end{array}$ & 60 & 32,1 & 26 & 13,1 & 122 & 91,0 & \\
\hline
\end{tabular}




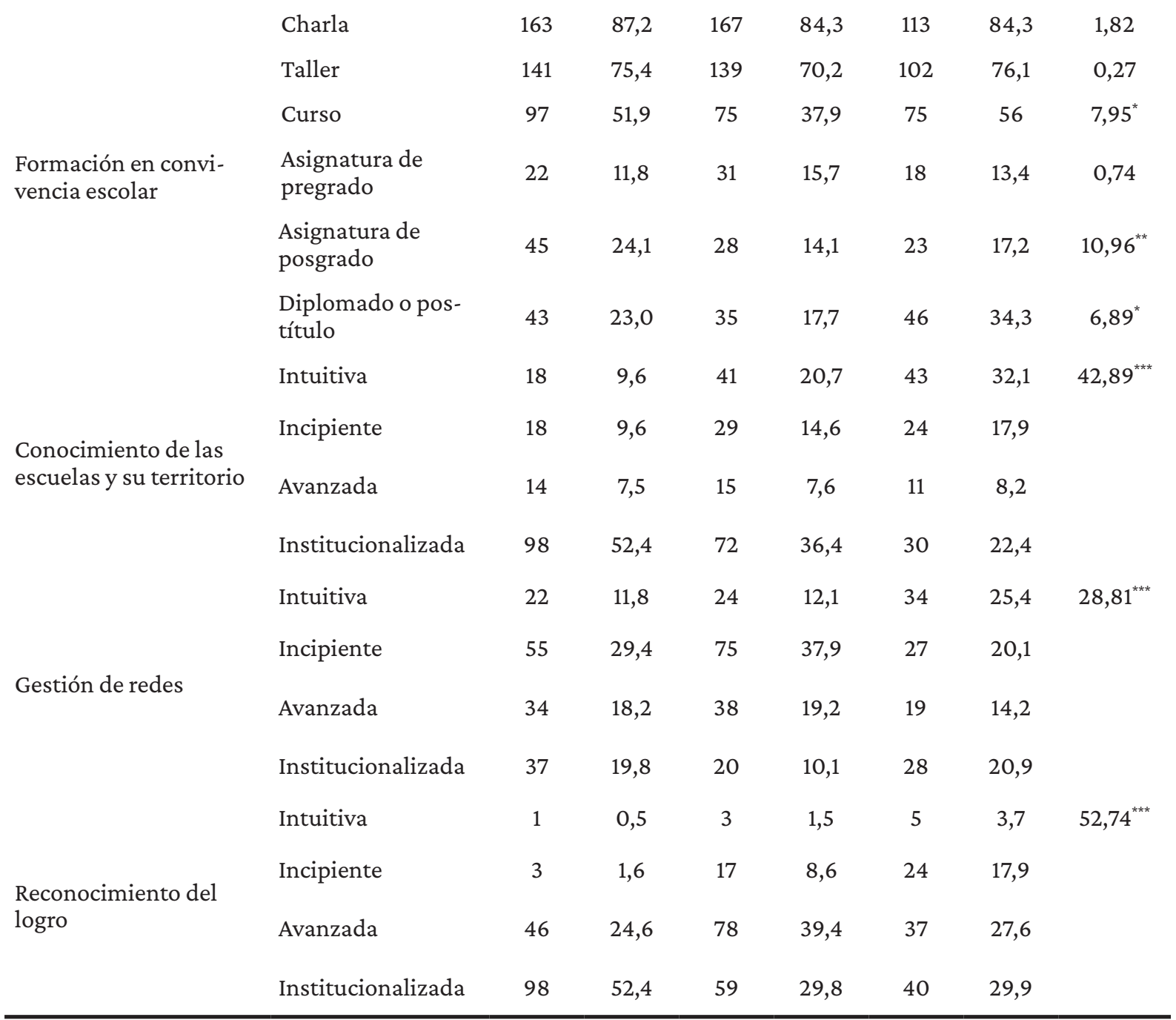

Nota. ${ }^{* * *} \mathrm{p}<0,001 ;{ }^{* *} \mathrm{p}<0,01 ;{ }^{*} \mathrm{p}<0,05$.

En la siguiente figura se observa la representación gráfica de árbol de problemas con el algoritmo CHAID exhaustivo. Se indica el porcentaje total y la clasificación de la variable dependiente (clúster) para cada nodo. Existen 9 nodos terminales y una clasificación de 4 ramificaciones (Figura 1).

Los resultados muestran que el factor más importante fue la socialización de roles y funciones de los encargados de CE de los establecimientos, la que se ramifica en tres nodos. En primer lugar, el nodo 1 no presenta ramificaciones y está compuesto por aquellos sostenedores que están en desacuerdo o muy en desacuerdo con dicha afirmación, y el 71,1\% corresponde al clúster 3.

El nodo 2 está compuesto principalmente por sostenedores que están solo de acuerdo con dicha afirmación o bien presentan una postura neutral, donde el $61,5 \%$ pertenece a al clúster 2 . Este se ramifica en los nodos 4 y 5 divididos por el acuerdo con que la planificación de CE comunal toma en cuenta las necesidades de los establecimientos. Así, el nodo 4 se subdivide por el grado de autonomía para reasignar recursos financieros en CE, donde el 14,1\% indica que se encuentra en desacuerdo con esta afirmación y el $20,4 \%$ que se encuentra de acuerdo.

En el caso del nodo 3, compuesto por quienes están muy de acuerdos con que se han socializado los roles y funciones de los encargados de CE de los establecimientos, el 77,6\% pertenece al clúster 1 , y se subdivide en los clústeres 6 y 7 por el hecho de proporcionar información a los establecimientos sobre su gestión en CE. 
Figura 1

Pertenencia a clústeres según acciones de gestión en convivencia escolar a nivel intermedio (Árbol de decisiones)

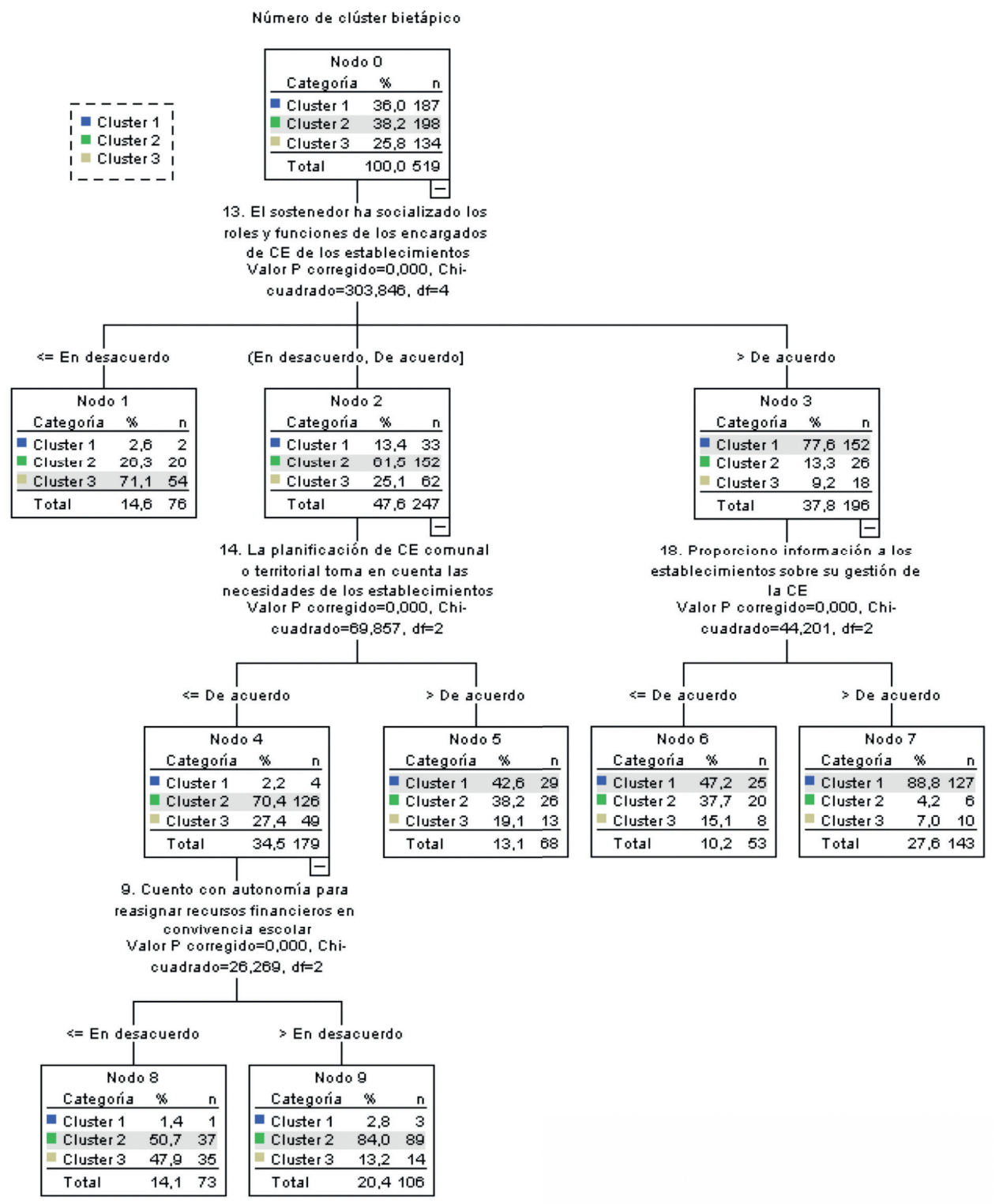

\section{Conclusiones}

La crisis mundial producida por el COVID-19 y los constantes movimientos político-sociales vividos en Chile durante los últimos años, han relevado la importancia de discutir los sentidos de la educación. Esta situación de crisis permanente ha permitido visualizar la función de la escuela en la provisión de servicios básicos en comunidades escolares altamente vulneradas, pero también, ha dilucidado una función de cuidado y contención (Kagawa, 2005; Tarabini, 2020). En este aspecto, la figura del nivel intermedio emerge como un eje articulador a nivel territorial, que permite implementar políticas contextualizadas a las características de sus comunidades escolares (Anderson et al., 2010).

En Chile, el nivel intermedio es complejo y diverso (Ascorra et al., 2020a) y durante los últimos años se les ha demandado responsabilizarse directamente con la mejora escolar, integrando elementos técnicos-pedagógicos y de aprendizaje transversal (Raczynski et al., 2019) como es el caso de la CE. Sin embargo, estas nuevas demandas no han venido acompañadas de políticas que orienten su quehacer, persistiendo una función administrativa (González et al., 2015; Pineda et al., 2020; Raczynski, 2012; Raczynski y Salinas, 2006; Raczynski et al., 2019; Román y Carrasco, 2007). 
Específicamente, la gestión de la CE a nivel intermedio ha sido un campo escasamente explorado (Ascorra et al.,, 2020a, 2020b; Gallardo, 2009a, 2009b; Sánchez et al., 2018). En este marco, es que el presente estudio se propuso identificar y caracterizar las modalidades de gestión de la CE en sostenedores chilenos, buscando ampliar los marcos interpretativos y de apoyo a estos equipos.

Los resultados de este estudio permiten identificar tres niveles de gestión de la CE a nivel intermedio. El primero de ellos, corresponde a un nivel de gestión de carácter intuitivo, donde se desarrollan escasas acciones promotoras de la CE. El segundo nivel, corresponde a una gestión en fase incipiente, donde se realizan parcialmente acciones promotoras de la CE, sin un foco claro. Y finalmente, un tercer nivel que corresponde a una gestión institucionalizada, donde se desarrollan diferentes acciones de gestión que promueven el desarrollo de la CE desde una perspectiva institucional, alineada a nivel horizontal y vertical, y con recursos financieros, materiales y humanos exclusivos para el área.

Estos grupos presentan ciertas particularidades entre sí. Los sostenedores en un nivel institucionalizado tienen en su mayoría una escuela a su cargo, lo que podría favorecer el reconocimiento de las necesidades de su comunidad escolar. Según Anderson y otros (2010) el nivel intermedio requiere contar con mecanismos de evaluación que permitan tener un conocimiento acabado de la realidad de sus escuelas. Esta información permite al nivel intermedio tener una línea base con la cual adecuar estrategias y políticas (NSBA, 2017) a la realidad de sus diferentes escuelas. Particularmente en Chile, Gallardo (2009a) y posteriormente Sánchez y otros (2018) evidenciaron que los SM -quienes poseen más escuelas a su cargo- presentan dificultades para reconocer y abordar las necesidades de sus escuelas. Estos estudios han expuesto que la cantidad y diversidad de escuelas a su cargo, y la escasez de recursos materiales y humanos son factores que afectan negativamente el abordaje de la convivencia escolar a nivel intermedio, impactando directamente en las acciones desarrolladas por los profesionales que gestionan la convivencia a nivel intermedio y en las escuelas.

Por otro lado, aquellos sostenedores en un nivel institucionalizado en la gestión de la convivencia escolar se percibieron con un mejor conocimiento del territorio y un mayor reconocimiento del logro. Estos resultados se condicen con la literatura internacional que refiere al conocimiento del territorio como un elemento crucial para la implementación de políticas, planes y programas contextualizados y participativos, que permitan aumentar los niveles de logro y mejora escolar (Anderson et al., 2010; Kull et al., 2016; NSBA, 2017). En Chile, Ascorra y otros (2020a) evidenciaron que el tamaño del equipo, las horas de contratación y la capacitación en CE tiene un efecto sobre la percepción del conocimiento del territorio, reconocimiento del logro y gestión de redes en convivencia escolar, demostrando que existen factores institucionales que repercuten en las acciones desarrolladas por el sostenedor.

Respecto a lo anterior, a pesar de que los participantes de este estudio mostraron en su mayoría no tener una formación especializada en CE a nivel de postgrado, aquellos sostenedores con una gestión institucionalizada mostraron un mayor porcentaje de profesionales con este tipo de formación. Estos resultados son relevantes considerando el nivel de especialización de los profesionales que gestionan la convivencia escolar. Actualmente, en Chile no existe ninguna legislación que defina un perfil profesional de quienes deben gestionar la convivencia. La Ley sobre Violencia Escolar, promulgada el año 2011 solo indicó la necesidad de contar con un encargado de convivencia escolar por escuela, sin definir un perfil profesional, recursos, ni cantidad de horas para desempeñar este rol (Ley No. 20.536, 2011). De hecho, a nivel intermedio no existe ninguna regulación que determine la existencia de equipos de apoyo para esta área. Considerando las múltiples demandas que han emanado de las nuevas políticas en Chile, muchos sostenedores se han visto en la necesidad de contar con apoyos "especializados" que permitan gestionar al menos administrativamente la convivencia escolar. Sin embargo, algunos estudios han develado una escasa especialización, y conformación de equipos con profesionales con una escasa experiencia (Ascorra et al., 2019b; Gallardo, 2009). A partir de estos antecedentes, es que consideramos necesario que futuras políticas puedan apoyar con orientaciones más claras a este nivel, pues -como hemos constatado previamente-, han ampliado significativamente sus funciones sin haberse otorgado los apoyos necesarios para fortalecer la implementación de estos nuevos roles.

Otro aspecto relevante en la conformación de los niveles de gestión fue la dependencia administrativa. En este aspecto, a pesar de que la dependencia municipal se muestre en su mayoría en un nivel intuitivo, la composición de sus escuelas es mucho más compleja que en la dependencia subvencionada. Los SM no solo poseen mayor cantidad y diversidad de establecimientos a su cargo, sino que también son responsables de un mayor porcentaje de estudiantes de nivel socioeconómico bajo, con necesidades educativas especiales, 
migrantes, entre otras características (Donoso y Arias, 2012; García y López, 2019; González, 2017; Jiménez et al., 2017; Santos y Elacqua, 2016; Valenzuela et al., 2010), lo que complejiza las posibilidades de desarrollar planes o programas que representen a la totalidad de sus escuelas.

Estos hallazgos ponen en evidencia el desbalance del sistema educacional chileno, ya que los sostenedores enfrentan situaciones disímiles; no obstante ello, deben responder a estándares homogéneos. Como podemos hipotetizar, el nivel de complejidad de gestionar la convivencia escolar en un solo establecimiento no es comparable con aquel sostenedor que debe gestionar la convivencia de todas las escuelas de su comuna (en algunos casos incluso más de 40 escuelas), las que además integran una matrícula más diversa y compleja a nivel psicosocial (Rodríguez et al., 2020; Córdoba et al., 2020). Además, como se ha evidenciado en estudios previos, las políticas educativas presionan de manera diferenciada, siendo los SM quienes presentan mayor cantidad de presiones y escasos apoyos (Ascorra et al., 2019; Bertoglia et al., 2011). Por lo tanto, la inequidad educativa no sólo se encuentra a nivel de escuela, como señala la literatura (Rodríguez et al., 2020; Santos y Elaqcua, 2016), sino que también se encuentra a nivel de gestión del nivel intermedio.

El contexto de pandemia ha ayudado a aumentar la brecha de desigualdad entre escuelas privadas, subvencionadas y municipales en Chile (Garrido, 2020; Murillo y Duk, 2020; Pávez et al., 2020; Quiroz, 2020). Sabemos que los estudiantes de escuelas municipales tienen menos acceso a internet, cuentan con menos tecnología de conectividad (computador, celular), cuentan con menos espacios en la casa que les permita el trabajo escolar (mayor hacinamiento y menos metros cuadrados), pero también cuentan con menos institucionalidad estatal reflejada en el sostenedor, en comparación con los estudiantes de escuelas subvencionadas y privadas. Este hecho, dificulta atender de manera seria y rigurosa las necesidades socioemocionales de una amplia gama de niños, niñas y jóvenes, lo cual puede afectar de manera sustancial sus trayectorias escolares. En este aspecto, consideramos que el soporte que pueda brindar el nivel intermedio o sostenedor resulta esencial, pues puede proveer de los apoyos necesarios a sus escuelas para aminorar las brechas generadas en contextos de crisis, las cuales sabemos que afectan de manera directa a los estudiantes más vulnerables (Murillo y Duk, 2020; Pavez et al., 2020).

A modo general, los resultados de este estudio permiten relevar la importancia de contar con modelos de gestión claros a nivel intermedio, que permitan propiciar el desarrollo y mejora de sus escuelas. Más aun en situaciones de crisis, donde el sostenedor puede actuar como facilitador u obstaculizador en la entrega de apoyos (Román y Carrasco, 2007). Asimismo, se hace necesario poder avanzar en políticas y programas descentralizados que favorezcan el reconocimiento y abordaje de las particularidades de las escuelas y del territorio. Las inequidades territoriales requieren ser abordadas a través de la descentralización, pues permite una mejor respuesta a realidades tan heterogéneas como las que se presentan en nuestro país (Quiroz, 2020).

Actualmente en Chile contamos con un sistema de administración escolar que, en un primer momento, permitió desconcentrar la gestión de recursos financieros y humanos del nivel central al nivel local. No obstante, muchas de las decisiones respecto del quehacer pedagógico persisten en el nivel central, restringiendo autonomía a los sostenedores. Incluso, muchos de los programas de apoyo a las escuelas, son delegados al nivel local, sobre demandando a los sostenedores con su administración (Pineda et al., 2020). En consecuencia, se observa una doble dependencia, que dificulta las posibilidades adecuar planes, programas y recursos a las necesidades locales (Donoso-Díaz y Benavides-Moreno, 2017).

Por último, este estudio permite visualizar algunas acciones que pueden constituirse como "claves" para la gestión de la CE. En primer lugar, vuelve a constatarse la necesidad de socializar los roles y funciones de los encargados de CE. Esto es coherente con estudios previos (Gallardo, 2009a; Sánchez et al., 2018), que refieren la importancia de asignar responsables en CE y definir sus funciones y tareas. No obstante, esta definición de "encargados" debe realizarse sin responsabilizar de forma exclusiva a estos por la gestión de la CE.

En segundo lugar, los sostenedores deben contar con personal especializado a nivel intermedio en temáticas vinculadas a la convivencia escolar. El contar con personal especializado permite un mejor afrontamiento a las necesidades de los profesionales de las escuelas y por consecuencia atender de mejor manera a las necesidades psicosociales de estudiantes y sus familias (Gallardo, 2009; Sánchez et al., 2018). Como se ha visualizado en estudios previos, esto marca la diferencia en la gestión de la convivencia escolar (Ascorra et al., 2020)

En tercer lugar, resulta imperativo que las políticas proporcionen los apoyos necesarios para aquellos sostenedores que cuentan con más escuelas a su cargo, pues -como se expuso anteriormente- presentan niveles 
de complejidad mayor para su gestión. La reciente Ley de Nueva Educación Pública (Ley No. 21.040, 2017), responsabiliza a los Servicios Locales de Educación de una mayor cantidad de escuelas -considerando aspectos pedagógicos y de aprendizaje transversal- confluyendo además distintos territorios, lo que representa un enorme desafío en la gestión del sostenedor. En este aspecto, se hace necesario contar con mecanismos y estrategias que permitan reconocer las particularidades de las escuelas y contar con modelos de gestión institucionalizados que permitan abordar de manera oportuna estas particularidades.

\section{Agradecimientos}

Este artículo es el resultado de una investigación financiada por el Centro de Investigación para la Educación Inclusiva (PIA-ANID CIE No. 160009) y la Pontificia Universidad Católica de Valparaíso a través del proyecto FONDECYT 1191883.

\section{Referencias}

Anderson, G. (2013). Cambios macropolíticos e institucionales, nuevos paradigmas e impacto en el trabajo docente. En M. Poggi (Comp.), Políticas docentes: Formación, trabajo y desarrollo profesional (pp. 73-120). Instituto Internacional de Planeamiento de la Educación/IIPE-UNESCO.

Anderson, S., Leithwood, K. y Strauss, T. (2010). Leading data use in schools: Organizational conditions and practices at the school and district levels. Leadership and Policy in Schools, 9(3), 292-327.

https://doi.org/10.1080/15700761003731492

Ascorra, P., Cárdenas, K. y Álvarez-Figueroa, F. (2020b). Gestión de la convivencia escolar a nivel intermedio: Diseño y validación de una escala. Revista Evaluar, 20(3), 1-19.

Ascorra, P., Cuadros, O., Cárdenas, K. y Meneses, J. (2020a). Autopercepción de la gestión de la convivencia escolar en sostenedores públicos chilenos. Psicoperspectivas, 19(1), 1-13.

https://doi.org/10.5027/psicoperspectivas-vol19-issue1-fulltext-1827.

Ascorra, P., Álvarez-Figueroa, F. y Queupil, J. P. (2019a). Managing school climate issues at the school district level: A comprehensive review of the literature. Universitas Psychologica, 18(5), 1-13.

https://doi.org/10.11144/Javeriana.upsy18-5.msci

Ascorra, P., López, V., Morales, M., Cuadros, O., Cárdenas, K. y Ortiz, S. (2019b). Caracterizar y fortalecer la gestión de la convivencia escolar en Chile. Centro de Investigación para la Educación Inclusiva.

Assaél, J., Cornejo, R., González, J., Redondo, J., Sánchez, R. y Sobarzo, M. (2011). La empresa educativa chilena. Educação e Sociedade, 32(115), 305-322. https://doi.org/10.1590/S0101-73302011000200004

Berlanga, V., Rubio, M. y Vila, R. (2013). Cómo aplicar árboles de decisión en SPSS. REIRE Revista d'Innovació i Recerca en Educació, 6(1), 65-79. https://doi.org/10.1344/reire2013.6.1615

Bertoglia, L., Raczynski, D. y Valderrama, C. (2011). Treinta años de política educativa descentralizada con efectos tardíos en calidad y equidad de la educación ¿Ausencia de enfoque territorial? RIMISP.

Campbell, C. y Fullan, M. (2006). Unlocking the potential for district-wide reform. Ontario Ministry of Education.

Canales, M. (2006). Metodologías de la investigación social. Lom Ediciones.

Carrasco, A., Bogolaski, F., Flores, C., Gutiérrez, G. y San Martín, E. (2014). Selección de Estudiantesy desigualdad en Chile: ¿Qué tan coactiva es la regulación que la prohíbe? FONIDE

Cohen, J., McCabe, L., Michelli, N. y Pickeral, T. (2009). School climate: Research, policy, teacher education and practice. Teachers College Record, 111(1), 180-213.

Córdoba, C., Laborda, A. y Reyes, C. (2020). Preferencias de Elección de Escuela en Dos Casos de Alta Segregación Escolar. REICE. Revista Iberoamericana sobre Calidad, Eficacia y Cambio en Educación, 18(4), 325-344.

https://doi.org/10.15366/reice2020.18.4.013 
Delannoy, F. y Guzmán, M. (2009). Experiencias internacionales en gestión descentralizada de la educación pública. En M. Marcel y D. Raczynski (Ed.), La asignatura pendiente: Claves para la revalidación de la educación pública de gestión local en Chile. CIEPLAN.

Donoso, S. y Arias, O. (2012). Distribución desigual de las oportunidades educativas en el territorio y migración de la matrícula escolar: El caso de la región de Los Lagos (Chile). Estudios Pedagógicos, 38(2), 35-54. https://doi.org/10.4067/S0718-07052012000200003.

Donoso-Díaz, S. y Benavides Moreno, N. (2017). Descentralización de la gestión de la educación pública e institucionalidad local en Chile: El caso de los directores comunales de educación. Innovar, 27(64), 115-128. https://doi.org/10.15446/innovar.v27n64.62372

Donoso-Díaz, S., Castro-Paredes, M, Alarcón-Leiva, J. y Davis-Toledo, G. (2015). Nudos críticos del sistema escolar chileno, hipótesis explicativas y propuestas para una nueva arquitectura del sector público. Ensaio: Avaliação e Políticas Públicas em Educação, 23(86), 37-71. https://doi.org/10.1590/S0104-40362015000100002.

Espínola, V. y Silva, M. E. (2009). Competencias del sostenedor para una efectiva gestión del mejoramiento educativo en el nivel local: Una propuesta. Expansiva.

Gallardo, G. (2009a). Gestión de la convivencia escolar desde sostenedores municipales: El nivel intermedio frente a los desafíos del presente. MINEDUC-UNICEF.

Gallardo, G. (2009b). Caso de estudio: Gestión de la convivencia escolar desde el nivel municipal. Sistematización y análisis de 5 experiencias en la región metropolitana. MINEDUC-UNICEF.

García, R. y López, V. (2019). Políticas de Educación Especial en Chile (2005-2015): Continuidades y cambios. Revista Brasileira de Educação Especial, 25(1), 1-16. https://doi.org/10.1590/s1413-65382519000100001

Garrido, M. (2020). Educar en tiempos de pandemia: Acentuación de las desigualdades en el sistema educativo chileno. Revista Caminhos da Educação: Diálogos, Culturas e Diversidades, 2(2), 43-68. https://doi.org/10.26694/caedu.v2i2.11241

González, R. (2017). Segregación educativa en el sistema chileno desde una perspectiva comparada. En Ministerio de Educación (Ed.), Ley de inclusión escolar (pp. 48-91). MINEDUC.

González, A., González, M. y Galdames, S. (2015). El sostenedor como agente de cambio: El rol de los coordinadores técnicos en el apoyo a establecimientos municipales chilenos. Pensamiento Educativo. Revista de Investigación Educacional Latinoamericana, 52(1), 47-64. https://doi.org/10.31619/caledu.n51.680

Hargreaves, A. (2010). Change from without: Lessons from other countries, systems, and sectors. En A. Hargreaves, A. Lieberman, M. Fullan y D. Hopkins (Eds.), Second international handbook of educational change (pp. 105-117). Springer Science+Business Media.

Hernández, R., Fernández, C. y Baptista, P. (2010). Metodología de la investigación. McGraw-Hill Interamericana.

Jiménez, F., Aguilera, M., Valdés, R. y Hernández, M. (2017). Migración y escuela: Análisis documental en torno a la incorporación de inmigrantes al sistema educativo chileno. Psicoperspectivas, 16(1), 105-116. https://doi.org/10.5027/psicoperspectivas-vol16-issue1-fulltext-940.

Kagawa, F. (2005). Emergency education: A critical review of the field. Comparative Education, 41(4), 487-503. https://doi.org/10.1080/03050060500317620

Kowalski, R. y Limber, S. (2007). Electronic bullying among middle school students. Journal of Adolescent Health, 41(6), 22-30. https://doi.org/10.1016/j.jadohealth.2007.08.017

Kull, R. M., Greytak, E. A., Kosciw, J. G. y Villenas, C. (2016). Effectiveness of school district antibullying policies in improving LGBT youths' school climate. Psychology of Sexual Orientation and Gender Diversity, 3(4), 407-415. https://doi.org/10.1037/sgd0000196

Ley No. 20.248. (1 de febrero de 2008). Establece Ley de Subvención Escolar Preferencial [Ley SEP]. Congreso de la República de Chile.

Ley No. 20.370. (2 de julio de 2010). Ley General de Educación [LGE]. Congreso de la República de Chile.

Ley No. 20.536. (17 de septiembre, 2011). Ley Sobre Violencia Escolar [LSVE]. Congreso de la República de Chile. 
Ley No. 20.845. (8 de junio de 2015). Ley de Inclusión Escolar [LIE]. Congreso de la República de Chile.

Ley No. 21.040. (24 de noviembre de 2017). Crea Nueva Educación Pública. Congreso de la República de Chile.

López, V., Cárdenas, K., Fernández-Vergara, A., González, L., Palacios, D., Ligueño, S. y Carrasco, C. (2020). Apoyos psicosociales: Avances y desafíos de las “duplas psicosociales SEP”. Centro de Investigación para la Educación Inclusiva. Pontificia Universidad Católica de Valparaíso.

Machel, G. (2001).The impact of war on children. UBC Press.

McIntyre, N. y Pigram, J. (1992). Recreation specialization reexamined: The case of vehicle-based campers. Leisure Research, 14, 3-15. https://doi.org/10.1080/01490409209513153

Ministerio de Educación de Chile. (2014). Estándares indicativos de desempeño para los establecimientos educacionales y sus sostenedores. MINEDUC.

Ministerio de Educación de Chile. (2015). Política nacional de convivencia escolar 2015-2018. MINEDUC

Ministerio de Educación de Chile. (2018). Directorio nacional de establecimientos. MINEDUC

Murillo, J. y Duk, C. (2020). El Covid-19 y las brechas educativas. Revista Latinoamericana de Educación Inclusiva, 14(1), 11-13. https://doi.org/10.4067/S0718-73782020000100011

National School Board Association. (2009). School climate guide for district policymakers and education leaders. NSBA.

National School Board Association (2017). Leading the change: Decision-making tools. NSBA.

Pardo, A. y Ruiz, M. A. (2005). Análisis de variables categóricas: El procedimiento Tablas de contingencia. En VVAA. Análisis de datos con SPSS 13 Base (pp. 207-234). McGraw-Hill/Interamericana.

Pavez, I., Poblete, D. y Galaz, C. (2020). Infancia migrante y pandemia en Chile: Inquietudes y desafíos. Sociedad e Infancia, 4, 259-262. https://doi.org/10.5209/soci.69619

Pineda, M., Palma, E., Assaél, J. y Redondo, J. (2020). Relaciones entre Coordinadores Municipales y Directores de Escuelas Públicas en Chile. REICE. Revista Iberoamericana sobre Calidad, Eficacia y Cambio en Educación, 19(1), 45-60. https://doi.org/10.15366/reice2021.19.1.003.

Quiroz, C. (2020). Pandemia COVID-19 e inequidad territorial: El agravamiento de las desigualdades educativas en Chile. Revista Internacional de Educación para la Justicia Social, 9(3e), 1-6.

Raczynski, D. (2012). Realidad de la educación municipal en Chile: ¿Liderazgo del sostenedor municipal? En J. Weinstein y G. Muñoz (Eds.), ¿Qué sabemos sobre los directores de escuela en Chile? (pp. 181-217). CEPPE.

Raczynski, D., Ribero, R. y Yáñez, T. (2019). Nivel intermedio del sistema escolar en Chile: Normativa y visión de los sostenedores acerca de las funciones, preparación para el cargo y las prácticas. Calidad en la Educación, 51, 382420. https://doi.org/10.31619/caledu.n51.680

Raczynski, D. y Salinas, D. (2006). La educación en el nivel local ¿Cómo manejar la tensión entre lo técnico y lo político? UNICEF.

Ramírez, C., Yáñez-Urbina, C. y Salinas, I. (2020). Chile, la democracia se acabó: Crisis institucional en el 18-O chileno. Re-Presentaciones. Investigación en Comunicación, 12, 6-28. https://doi.org/10.35588/rp.v0i12.4317

Reimers, F. y Schleicher, A. (2020). Un marco para guiar una respuesta educativa a la pandemia del 2020 del COVID-19. OECD-OEI.

Rodríguez, C., Espinosa, E. y Padilla, G. (2020). Dónde quiero que estudien mis hijos/as: caracterización de la oferta educativa y sus niveles de demanda en Chile. Revista de Estudios y Experiencias en Educación, 19(41), 57-70. https://doi.org/10.21703/rexe.20201941rodriguez4

Román, M. y Carrasco, A. (2007). Los niveles intermedios del sistema escolar chileno: posibilidades y limitaciones para la equidad. REICE. Revista Iberoamericana sobre Calidad, Eficacia y Cambio en Educación, 5(1), 1-21.

Rundle-Thiele, S., Kubacki, K., Tkaczynski, A. y Parkinson, J. (2015). Using two-step cluster analysis to identify homogeneous physical activity groups. Marketing Intelligence \& Planning, 33(4), 522-537.

https://doi.org/10.1108/MIP-03-2014-0050 
Sánchez, M., Vicuña, A. y Subercaseaux, J. (2018). Estudio sobre la gestión de la convivencia escolar entre los sostenedores de los establecimientos. MINEDUC.

Santos, H. y Elacqua, G. (2016). Segregación socioeconómica escolar en Chile: Elección de la escuela por los padres y un análisis contrafactual teórico. REVISTA CEPAL, 119, 133-148.

Tarabini, A. (2020). ¿Para qué sirve la escuela? Reflexiones sociológicas en tiempos de pandemia global. Revista de Sociología de la Educación, 13(2), 145-155. https://doi.org/10.7203/RASE.13.2.17135

UNESCO. (2020). Impacto de la COVID-19 en la educación. UNESCO.

Universidad de Chile. (2020). Salud mental en contexto de pandemia: Documento para Mesa Social COVID-19. Universidad de Chile.

Valenzuela, J. P., Bellei, C. y De los Rios, D. (2010). Segregación escolar en Chile. En S. Martinicy G. Elacqua (Eds.), ¿Fin de ciclo? Cambios en la gobernanza del sistema educativo (pp. 209-239). UNESCO.

Weinstein, J., Fuenzalida, A. y Muñoz, G. (2010). La subvención preferencial: Desde una difícil instalación hacia su institucionalización. En S. Martinic y G. Elacqua (Eds.), ¿Fin de ciclo? Cambios en la gobernanza del sistema educativo (pp. 161-282). UNESCO.

\section{Breve CV de los/as autores/as}

\section{Paula Ascorra Costa}

Doctora en Psicología de la Universidad de Chile. Investigadora Principal en la línea "Convivencia y Bienestar Escolar” del Centro de Investigación para la Educación Inclusiva (Proyecto PIA-ANID CIE160009). Académica de la Escuela de Psicología de la Pontificia Universidad Católica de Valparaíso. Investigadora Responsable FONDECYT (No. 1191883) cuyo foco es la convivencia escolar desde una perspectiva democrática. Presenta una amplia trayectoria de investigación en convivencia escolar, participando en diversos proyectos vinculados a la temática. Email: paula.ascorra@pucv.cl

ORCID ID: http://orcid.org/0000-0001-9449-8273

\section{Karen Cárdenas Mancilla}

Psicóloga de la Universidad Santo Tomás. Diplomada en Psicología educacional, Convivencia escolar y Metodologías de investigación en Ciencias Sociales de la Pontificia Universidad Católica de Valparaíso. Actualmente se desempeña como Investigadora en la línea de “Convivencia y Bienestar Escolar” del Centro de Investigación para la Educación Inclusiva (Proyecto PIA-ANID CIE160009) y como profesional de apoyo en un proyecto FONDECYT (No. 1191883). Profesor asociado de la Pontificia Universidad Católica de Valparaíso. Email: karen.cardenas@pucv.cl

ORCID ID: https://orcid.org/0000-0003-2828-6824

\section{Javier Torres Vallejos}

Psicólogo de la Universidad de Santiago de Chile y Doctor en Psicología por la Pontificia Universidad Católica de Valparaíso y por la Universitat de Girona, España. Académico y director del programa de "Magister en Educación Emocional y Convivencia Escolar” de la Universidad Andrés Bello. Investigador colaborador en la línea "Convivencia y Bienestar Escolar" del Centro de Investigación para la Educación Inclusiva (Proyecto PIA-ANID CIE160009). Investigador responsable del proyecto FONDECYT (No. 3210525), cuyo foco es el análisis de trayectorias educativas y bienestar subjetivo de estudiantes.

Email: javier.torres@unab.cl

ORCID ID: https://orcid.org/0000-0002-4229-7768 